\title{
Ambiguity in the evaluation of the effective action on the cone
}

\author{
Devis Iellici* \\ Dipartimento di Fisica, Università di Trento, and Istituto Nazionale di Fisica Nucleare, Trento, Italy \\ Edisom S. Moreira, Jr. ${ }^{\dagger}$ \\ Instituto de Física Teórica, Universidade Estadual Paulista, Rua Pamplona 145, 01405-900, São Paulo, São Paulo, Brazil
}

(Received 24 July 1998; published 24 November 1999)

\begin{abstract}
An ambiguity in the computation of the one-loop effective action for fields living on a cone is illustrated. It is shown that the ambiguity arises due to the noncommutativity of the regularization of ultraviolet and (conical) boundary divergencies. [S0556-2821(99)02122-0]

PACS number(s): 04.62.+v, 04.70.Dy
\end{abstract}

\section{INTRODUCTION}

Recently there has been much interest in computations of one-loop effects for quantum fields living in backgrounds with conical singularities, especially in the context of quantum corrections to the Bekenstein-Hawking entropy $[1,2]$. In this context, conical singularity methods [2-16] have been shown to be a powerful tool. There is, nevertheless, a controversy in the literature concerning two possible kinds of approaches to computing one-loop effects on the cone, leading to different results (for a review, see [17]).

In the local approach $[4,5,12]$, one starts regularizing and renormalizing local quantities, such as the effective Lagrangian density or the energy-momentum tensor. Global quantities, for example the effective action, are then obtained by integrating the local ones over the background. Local quantities show a nonintegrable singularity at $r=0$, where $r$ is the proper distance from the apex of the cone. For a massless field in $D$ dimensions, for example, simple dimensional considerations determine the dependence of the effective Lagrangian density on $r$ as being $r^{-D}$. Therefore, integration over the background usually requires the introduction of a cutoff at a proper distance $\epsilon$ from the tip of the cone.

In the global approach $[3,6,10,11,13,15,16]$, integration over the background is performed before ultraviolet regularization, resulting that the volume cutoff $\epsilon$ is not required. At this point it should be remarked that the local and global approaches are equivalent to each other when the background is a smooth manifold.

In the context of quantum fields at finite temperature in Rindler space-time (or near the horizon of a black hole), both approaches lead to a divergent entropy. However the origin of the divergences seems quite different-in the local approach the divergence is associated with the (divergent) local temperature on the horizon [18], contrasting with the ultraviolet (uv) nature of the divergence in the global approach. Another important difference concerns dependence on the temperature $T$ of the thermodynamical quantities $[19,12,14]$. In the local approach the free energy, at high temperatures, shows a leading contribution proportional to $T^{D}$, whereas in

\footnotetext{
*Electronic address: devis.iellici@telital.com

†Electronic address: moreira@cpd.efei.br
}

the global approach it behaves as $T^{2}$. Finally, in the global approach the divergences can be renormalized into the bare gravitational action $[2,10,11]$, whereas such a procedure is not possible in the context of the local approach [20].

Considering these facts, it can be said that there is an ambiguity in the computation of one-loop effects on the cone. As different uv regularization techniques have been used in the literature (for example, the $\zeta$ function procedure $[12,21]$ in the case of the local approach, and the Schwinger proper-time $[3,6,10,11,15,16]$ or the Pauli-Villars [9] procedures in the case of the global approach), it was not clear whether the origin of the ambiguity had to do with their different features or some other reason. In this paper we show that the origin of the ambiguity is not in the uv regularization employed (since it appears within any regularization scheme as will be shown), but that it arises from a conflict between regularization of uv and horizon divergencies. One may say, from the mathematical point of view, that the conflict is simply due to the fact that the uv regulator $\delta$ and the horizon cutoff $\epsilon$ appear as in $\epsilon^{2} / \delta$, and therefore the result depends radically on the order in which these regulators are sent to zero.

The next section illustrates the ambiguity by employing the Schwinger proper-time regularization. A discussion follows where we give a particle-loop interpretation to the two approaches and argue that the local approach seems to be supported by physical considerations. In Appendixes A and $\mathrm{B}$, the ambiguity is considered in the context of a pointsplitting regularization, and in a wide class of Schwinger-like regularizations, respectively.

\section{LOCAL VS GLOBAL APPROACH}

Let us see how the ambiguity arises. For simplicity a scalar field will be considered, but the argument is essentially the same for higher spins. The starting point is a representation of the local heat kernel for minus the Laplace-Beltrami operator $A=-\Delta_{\beta}$ on the four-dimensional flat cone $C_{\beta}$ $\times R^{2}$ with metric $d s^{2}=r^{2} d \tau^{2}+d r^{2}+d y^{2}+d z^{2}$, where $\tau$ $\in[0, \beta], r \in R^{+}$and $y, z \in R$. The deficit angle of the cone is $2 \pi-\beta$. In the context of finite temperature theory in the Rindler space-time (or near the horizon of a black hole), $\beta^{-1}$ is the temperature. A useful representation of the heat kernel is given by $[22-24,5]$ 
$K_{t}^{\beta}(x, x \mid A)=\frac{1}{16 \pi^{2} t^{2}}+\frac{i}{32 \pi^{2} \beta t^{2}} \int_{\Gamma} d w e^{-\left(r^{2} / t\right) \sin ^{2}(w / 2)} \cot \frac{\pi w}{\beta}$,

where the contour $\Gamma$ consists of two branches intersecting the real axis very close to the origin. One branch goes from $-\pi+i \infty$ to $-\pi-i \infty$, and the other one from $\pi-i \infty$ to $\pi$ $+i \infty$. An advantage of this representation is that the flatspace heat kernel (the first term on the right-hand side) appears isolated from the conical singularity contribution. This feature will show clearly that the ambiguity arises due to the presence of a conical singularity.

The local heat kernel (1) can be integrated over the background to yield the well-known integrated heat kernel [25]

$$
K_{t}^{\beta}(A)=\frac{\mathcal{A}_{H} V\left(C_{\beta}\right)}{16 \pi^{2} t^{2}}+\frac{\mathcal{A}_{H}}{48 \pi t}\left(\frac{2 \pi}{\beta}-\frac{\beta}{2 \pi}\right),
$$

where $V\left(C_{\beta}\right)=\beta R^{2} / 2$ is the (infinite) area of a cone of radius $R$, and $\mathcal{A}_{H}$ is the (infinite) area of the transverse dimensions - the horizon area. The above integration has been performed by using formulas computed by Dowker in [23] (see also [24]). Defining

$$
C_{2 n}(\beta):=\frac{i}{4 \beta} \int_{\Gamma} d w \cot \left(\frac{\pi w}{\beta}\right)\left(\sin ^{2} \frac{w}{2}\right)^{-n},
$$

one has, for $n=1,2$,

$$
\begin{gathered}
C_{2}(\beta)=\frac{\pi}{3 \beta}\left(\frac{2 \pi}{\beta}-\frac{\beta}{2 \pi}\right), \\
C_{4}(\beta)=\frac{1}{15} C_{2}(\beta)\left[\left(\frac{2 \pi}{\beta}\right)^{2}+11\right] .
\end{gathered}
$$

Let us focus on the computation of the one-loop effective action for a neutral massless scalar field on the cone (the massive case is treated in Appendix A), noting that the ambiguity should also be present in the computation of other global quantities. The effective action is determined by integrating the effective Lagrangian over the background

$$
W_{\mathrm{eff}}=\int d^{4} x \sqrt{g} L_{\mathrm{eff}}(x)
$$

Since, from dimensional considerations, it is expected that $L_{\text {eff }}(x)$ behaves as $r^{-4}$, in the integration over $r$ above one introduces a cutoff at a distance $\epsilon$ from the horizon ( $r$ $=0$ ). The effective Lagrangian can be computed by integrating over the proper-time $t$ the product of the local heat kernel by $t^{-1}$, and since it is uv divergent some regularization procedure is required. Here we will apply the Schwinger regularization which is widely used in the literature. Note however that the results hold for any regularization procedure (see the Appendixes).

According to the Schwinger regularization procedure, an infinitesimal uv cutoff $\delta$ is introduced in the integration over $t$

$$
L_{\mathrm{eff}}(x)=-\frac{1}{2} \int_{\delta}^{\infty} \frac{d t}{t} K_{t}^{\beta}(x, x \mid A),
$$

resulting in

$$
W_{\mathrm{eff}}=-\frac{\beta \mathcal{A}_{H}}{2} \int_{\epsilon}^{\infty} r d r \int_{\delta}^{\infty} \frac{d t}{t} K_{t}^{\beta}(r \mid A) .
$$

By using Eq. (1), one integrates over $t$ and $r$ (note that if the regulators $\delta$ and $\epsilon$ are not set equal to zero in an early stage, the order in which the integrations are performed is not relevant), resulting in

$$
\begin{aligned}
W_{\mathrm{eff}}= & -\frac{\mathcal{A}_{H} V\left(C_{\beta}\right)}{64 \pi^{2} \delta^{2}}+\frac{i \mathcal{A}_{H}}{128 \pi^{2} \epsilon^{2}} \\
& \times \int_{\Gamma} d w \frac{\cot \frac{\pi w}{\beta}}{\sin ^{4} \frac{w}{2}}\left(e^{-\left(\epsilon^{2} / \delta\right) \sin ^{2}(w / 2)}-1\right) .
\end{aligned}
$$

Clearly as the regulators appear in the ratio $\epsilon^{2} / \delta$, the (regularized) effective action depends on the order in which the regulators are sent to zero. Note that the first term on the right-hand side of Eq. (5) encodes essentially the flat-space ultraviolet divergence (which can be renormalized by redefining the bare cosmological constant), whereas the second term encodes the conical singularity contribution. Now, noticing that if the horizon cutoff $\epsilon$ is kept fixed then the conical contribution in Eq. (5) is uv finite, and observing Eqs. (3) and (4), when $\delta \rightarrow 0$ one is led to (up to $e^{-\epsilon^{2} / \delta}$ terms)

$$
W_{\mathrm{eff}}=-\frac{\mathcal{A}_{H} V\left(C_{\beta}\right)}{64 \pi^{2} \delta^{2}}-\frac{\beta \mathcal{A}_{H}}{32 \pi^{2} \epsilon^{2}} C_{4}(\beta)
$$

Apart from the uv divergent term (which is irrelevant, since it can be renormalized away) this result is exactly that obtained in the local approach by means of local $\zeta$ function [12], dimensional [20], and point-splitting regularization (see Appendix A). Interpreting $W_{\text {eff }} / \beta$ as a free energy, the Planckian term $\left(1 / \beta^{4}\right)$ reproduces precisely the one in the result by Susskind and Uglum [2], which was obtained by counting eigenmodes and employing a WKB approximation. There is also agreement with respect to the mass corrections $\left(1 / \beta^{2}\right)$ (see Appendix A and [17]). Nevertheless, Eq. (6) leads to a non-Planckian contribution $\left(1 / \beta^{2}\right)$ in the free energy, which is absent in [2]. The source of this discrepancy is not well understood as yet, but it seems to be related to the presence of a conical singularity in the procedures yielding Eq. (6), and to the very interpretation of $W_{\text {eff }} / \beta$ as a free energy in the present context (see [26], and references therein). Note that according to Eq. (6), uv divergences affect only the temperature-independent contribution in the corresponding free energy, as usually is the case.

The global approach corresponds to removing the horizon cutoff $(\epsilon \rightarrow 0)$ from Eq. (5) keeping the uv regulator $\delta$ fixed, resulting in 


$$
W_{\text {eff }}=-\frac{\mathcal{A}_{H} V\left(C_{\beta}\right)}{64 \pi^{2} \delta^{2}}-\frac{\beta \mathcal{A}_{H}}{32 \pi^{2} \delta} C_{2}(\beta),
$$

which is in agreement with early results $[3,6,10,11]$.

There are some important remarks to be made concerning the dependence of the results on the regularization procedure employed. In the local approach the conical contribution in $W_{\text {eff }}$ is independent of the regularization employed (see the Appendixes). This is not the case in the global approach, which does depend on the regularization procedure. As is shown in Appendix B, in the context of the global approach, the conical contribution in $W_{\text {eff }}$ vanishes in both $\zeta$ function and dimensional regularizations, whereas it consists of an uv divergent term according to the Schwinger regularization procedure. There is no contradiction though, once one realizes that the divergent term is proportional to the SeeleyDeWitt coefficient $A_{1}=\beta \mathcal{A}_{H} C_{2}(\beta)$ [see Eq. (2)], and therefore can be renormalized away by redefining the bare Newton constant in the gravitational action [2,9-11]. In the local approach no such renormalization is possible, since the conical contribution is not proportional to geometrical terms which can be found in the gravitational action. This is consistent with the statement above that, in the local approach, all regularizations lead to the same result. These facts illustrate the different physical nature of the local and global results. In the context of the computation of quantum corrections to the Bekenstein-Hawking entropy, it follows that in the global approach the entropy remains in the form $S$ $=\mathcal{A}_{H} / 4 G$, which is finite even after quantum corrections have been considered, provided that $G$ is the renormalized Newton constant [2]. In the local approach, on the contrary, quantum corrections result in a divergent total entropy $[1,18]$.

\section{DISCUSSION}

It is clear that the ambiguity considered in this paper arises due to the fact that the order in which uv and horizon divergences are isolated (regularized) matters. Let us discuss the subject further.

According to a path integral interpretation associated with the heat kernel, the one-loop effective action is obtained by summing over all particle loops. In the local approach, one considers a particular point and the one-loop contribution to the effective Lagrangian is obtained by summing over all loops starting and ending at that point. The effective action is then obtained by integrating the effective Lagrangian over the space. In the global approach, instead, one sums over all possible closed loops without having to consider local quantities.

In this scenario, uv divergences originate from infinitesimal loops about to contract to a point. There are two kinds of loops on a cone. Loops that do not wind around the conical singularity probe only flat-space. Infinitesimal loops of this kind give essentially the usual uv divergence contributions in flat space, which is the same in both approaches. They correspond to the first term on the right-hand side of Eqs. (6) and (7). Loops of the other kind are those that wind around the conical singularity. They detect the conical singularity and give rise to the second terms on the right-hand side of Eqs. (6) and (7). In the local approach, these loops cannot be contracted to a point and so they do not give ultraviolet divergences in the local quantities (they are responsible for the conical part in Eq. (6) which is, in fact, uv finite). However, by considering points closer and closer to the apex of the cone, one picks up contributions corresponding to smaller and smaller loops encircling the singularity, and which give rise to the divergence as $\epsilon \rightarrow 0$. In the global approach, on the contrary, by summing over all loops one considers also infinitesimally small loops winding around the singularity. Thus an uv divergent contribution, which depends on the deficit angle, arises in Eq. (7). It is then clear that horizon divergences in the local approach and uv divergences in the global approach are different manifestations of the same phenomenon.

At this point an inescapable question is posed: which one of these conical contributions is the correct one? Mathematically, the origin of the ambiguity is clear, resulting that both approaches seem to be equally correct. Therefore the answer to the question above should come from physical arguments. From this point of view the result given by the local approach seems to be supported by the fact that, before determining global quantities, one would like to know corresponding local quantities (such as the energy-momentum tensor, for example). In order to be meaningful, these local quantities should have their uv divergences appropriately regularized. It is important to stress that no such calculation is possible in the context of the global approach, since uv regularization is performed only after integration over the background.

There are also some facts supporting the local approach. Statistical mechanics models for quantum fields vibrating near the horizon, e.g., the brick wall model $[1,2]$, fix a Planckian behavior for the free energy $\left(1 / \beta^{4}\right)$. As mentioned previously, the corresponding local approach result fits this behavior, at least at high temperatures. According to the global approach the free energy has a non-Planckian behavior $\left(1 / \beta^{2}\right)$. Furthermore one expects the divergence in the free energy to be related with the divergence in the local temperature on the horizon [18]. This correspondence holds in the local approach result.

Before closing, we would like to comment on some previous works $[17,27,28]$. The authors of [17] mention that the local approach (roughly speaking local and global approaches correspond, respectively, to "volume cutoff" and "ultraviolet limit" in the terminology of [17]) does not correspond to a complete theory on the cone for the following fact. Since the integration over $r$ stops at an infinitesimal distance $\epsilon$ from the horizon ( $r=0)$, and the quantities in the local approach carry the cutoff $\epsilon$, in the local approach one is in fact working in an "incomplete background"zon (apex of the cone) is missing. That is not quite correct. In this paper we have shown that in both approaches one uses the same heat kernel, which is well defined on the whole cone and built up with eigenmodes which are required to be finite on the horizon. By stopping the integration over $r$ at a certain distance from the horizon one is simply isolating 
the horizon divergences, and not truncating the cone as the authors of [17] seem to suggest.

Entropy corresponding to the effective actions considered in this paper is often identified (for $\beta=2 \pi$ ) as quantum correction to the Bekenstein-Hawking entropy of a Schwarzschild black hole. However we should recall that such effective actions have been obtained in Rindler spacetime. When compared with results obtained by working in black hole background itself (see, for example, [27] where WKB approximation is applied in the context of the brick wall model [1], leading to an expression for the effective action involving the Epstein-Hurwitz $\zeta$ function), they present the correct leading divergence on the black hole horizon [see, e.g., Eq. (A8)], but fail to reproduce a massless logarithmic (subleading) divergence which arises due to the nontrivial topology of the black hole [29]. In semiclassical approaches topology manifests itself when integration over the background is performed [30].

It would be worth investigating the issues considered here in the context of the approach in [28], where a method for determining heat kernel involving a partial resummation of the Schwinger-DeWitt series is presented.

\section{ACKNOWLEDGMENTS}

D.I. is grateful to Emilio Elizalde, Valter Moretti, and, in particular, Sergio Zerbini for useful discussions and sugges- tions. E.M. is grateful to George Matsas for valuable discussions and to FAPESP (through Grant No. 96/12259-1) for financial support.

\section{APPENDIX A: POINT-SPLITTING PROCEDURE}

In this appendix we show how the ambiguity arises when a point-splitting regularization procedure is used.

In the following we sketch the evaluation of

$$
L_{\mathrm{eff}}(x)=-\frac{1}{2} \lim _{x^{\prime} \rightarrow x} \int_{0}^{\infty} \frac{d t}{t} K_{t}^{\beta}\left(x, x^{\prime} \mid A\right)
$$

in an $N$ dimensional cone and then the associated effective action. The heat kernel for a massive scalar field can be read from the integrand of the proper-time representation of the Feynman propagator in [31],

$K_{t}^{\beta}(\Delta)=\frac{2 \pi}{\beta(4 \pi t)^{N / 2}} e^{-r^{2} / 2 t-m^{2} t} \sum_{n=-\infty}^{\infty} I_{2 \pi|n| / \beta}\left(r^{2} / 2 t\right) e^{i 2 \pi n \Delta / \beta}$.

Here $\Delta:=\tau-\tau^{\prime}$ and the coordinates of points $x$ and $x^{\prime}$ have correspondingly been identified, except the angular coordinates. Using [32] one finds

$$
\begin{aligned}
\int_{0}^{\infty} \frac{d t}{t} K_{t}^{\beta}(\Delta)= & \frac{2 \pi}{\beta\left(2 \pi^{1 / 2}\right)^{N} r^{N}} \times \sum_{n=-\infty}^{\infty} e^{i 2 \pi n \Delta / \beta}\left\{\frac{\Gamma\left[N / 2+\nu_{n}\right] \Gamma[(1-N) / 2]}{\pi^{1 / 2} \Gamma\left[(2-N) / 2+\nu_{n}\right]} \times{ }_{1} F_{2}\left[\frac{1-N}{2} ; \frac{2-N}{2}-\nu_{n}, \frac{2-N}{2}+\nu_{n} ;(m r)^{2}\right]\right. \\
& \left.+2^{-2 \nu_{n}}(m r)^{N+2 \nu_{n}} \frac{\Gamma\left[-N / 2-\nu_{n}\right]}{\Gamma\left[1+\nu_{n}\right]} \times{ }_{1} F_{2}\left[\frac{1}{2}+\nu_{n} ; \nu_{n}+\frac{N+2}{2}, 1+2 \nu_{n} ;(m r)^{2}\right]\right\},
\end{aligned}
$$

where $\nu_{n}:=2 \pi|n| / \beta$ and ${ }_{1} F_{2}[a ; b, c ; z]$ denotes the generalized hypergeometric function [33] which converges for all values of $z$. Keeping mass corrections in Eq. (A3) up to second order in $m r$, Eq. (A1) yields

$$
\begin{aligned}
L_{\text {eff }}(r)= & \frac{i}{(1-N) r^{2}} \lim _{\Delta \rightarrow 0}\left[\partial_{\Delta}^{2}+\left(\frac{N-2}{2}\right)^{2}\right. \\
& \left.+\frac{1-N}{2}(m r)^{2}\right] D_{\mathcal{F}}(\Delta),
\end{aligned}
$$

where $D_{\mathcal{F}}(\Delta)$ is the massless Feynman propagator [31,34]. When $N=4$, expansion of $D_{\mathcal{F}}(\Delta)$ in powers of $\Delta$ is given by

$$
D_{\mathcal{F}}(\Delta)=\frac{i}{8 \pi^{2} r^{2}}\left(-\frac{2}{\Delta^{2}}-\frac{2 \pi^{2}}{3 \beta^{2}}-\frac{2 \pi^{4}}{15 \beta^{4}} \Delta^{2}+\mathcal{O}\left(\Delta^{3}\right)\right)
$$

Inserting Eq. (A5) in Eq. (A4), the ultraviolet divergences in the limit $\Delta \rightarrow 0$ may be eliminated by subtracting the contribution corresponding to some particular $\beta=\beta_{o}$,

$$
L_{\mathrm{eff}}(r)=\frac{1}{24 \pi^{2} r^{4}}\left[f(\beta)-f\left(\beta_{o}\right)\right]
$$

with $f(\beta):=-4 \pi^{4} / 15 \beta^{4}-2 \pi^{2} / 3 \beta^{2}+\pi^{2}(m r)^{2} / \beta^{2}$. The effective action is obtained by integrating the effective Lagrangian over the background

$$
W_{\mathrm{eff}}=\mathcal{A}_{H} \int_{0}^{\beta} d \tau \int_{\epsilon}^{R} d r r L_{\mathrm{eff}}(r)
$$

where $\epsilon$ and $R$ are the horizon and volume cutoffs, respectively. Finally replacing Eq. (A6) in Eq. (A7) one finds 


$$
W_{\text {eff }}=-\frac{\mathcal{A}_{H} \pi^{2}}{180 \epsilon^{2} \beta^{3}}-\frac{\mathcal{A}_{H}}{72 \epsilon^{2} \beta}-\frac{\mathcal{A}_{H} m^{2}}{24 \beta} \ln m \epsilon+C_{\beta_{o}} \beta,
$$

where $C_{\beta_{o}}$ denotes a constant which ensures that $W_{\text {eff }}=0$ when $\beta=\beta_{o}$. In Eq. (A8) the upper cutoff contribution has been omitted.

When $m=0$, Eq. (A8) is in agreement with Eq. (6), and the mass correction is in agreement with that computed by other methods [35,21]. It is worth remarking that the result above differs from that obtained by Dowker [4], where the expectation value of the energy density is integrated over the background to give the internal energy. The difference is in the non-Planckian massless term in the corresponding free energies. As mentioned previously, there has not appeared yet a satisfactory explanation for these low temperature discrepancies (see $[26,20]$ ).

If in Eq. (A7), by setting $\epsilon=0$ and $R=\infty$, the integration over the background is performed before the integration over $t$, then one is led to the global approach result [7]. Note that in this case the splitting $\Delta=\tau-\tau^{\prime}$ is not enough to handle uv divergences, and a further regulator (e.g., the proper-time cutoff $\delta$ ) is required.

\section{APPENDIX B: GENERALIZED SCHWINGER REGULARIZATION}

This appendix considers the ambiguity in the context of a wide class of Schwinger type regularizations. In general the effective action can be computed from the heat-kernel as follows (see, e.g., [36-38])

$$
W_{\mathrm{eff}}=-\frac{1}{2} \lim _{\delta \rightarrow 0} \int_{0}^{\infty} \frac{d t}{t} \rho(\delta, t) K_{t}(A),
$$

where the regularizing function $\rho(\delta, t)$ has to satisfy some requirements. For example, it is assumed that $\lim _{\delta \rightarrow 0} \rho(\delta, t)$ $=1$ and that (for sufficiently large $\delta$ ) $\rho(\delta, t)$ has to regular- ize the divergence at $t=0$. Among possible regularizing functions one has the proper-time cutoff, $\rho(\delta, t)=\Theta(t-\delta)$ (considered previously in this work), $\zeta$ function, $\rho(\delta, t)$ $=(d / d \delta)\left[t^{\delta} / \Gamma(\delta)\right]$, dimensional regularization, $\rho(\delta, t)$ $=(4 \pi t)^{-\delta}$, and Pauli-Villars regularization, $\rho(\delta, t)=(1$ $\left.-e^{-t / \delta}\right)^{3}$ (in four dimensions). Effective actions computed using different regularizing functions differ by terms which are divergent as $\delta \rightarrow 0$, but proportional to the Seeley-DeWitt coefficients $A_{0}, A_{1}$, and $A_{2}$. Therefore these terms can be renormalized away by redefining the bare coupling constants in the gravitational action [39].

Let us then consider a cone as background. Employing an arbitrary regularizing function (flat-space contributions, which can be renormalized away, will be dropped) Eq. (5) becomes

$$
W_{\mathrm{eff}}=-\frac{i \mathcal{A}_{H}}{128 \pi^{2}} \int_{\Gamma} d w \frac{\cot \frac{\pi w}{\beta}}{\sin ^{2} \frac{w}{2}} \int_{0}^{\infty} \frac{d t}{t^{2}} \rho(\delta, t) e^{-\left(\epsilon^{2} / t\right) \sin ^{2}(w / 2)} .
$$

In the local approach one sets $\delta \rightarrow 0$, so that $\rho(\delta, t) \rightarrow 1$. After integrating over $t$ one is left with Eq. (6), whichever regularizing function one chooses. In the global approach, on the other hand, one sets $\epsilon \rightarrow 0$ first, so that

$$
W_{\mathrm{eff}}=-\frac{\beta \mathcal{A}_{H}}{32 \pi^{2}} C_{2}(\beta) \int_{0}^{\infty} \frac{d t}{t^{2}} \rho(\delta, t)
$$

The integral over $t$ depends on the regularizing function. It is divergent in some regularizations (proper-time cutoff, PauliVillars regularizations) and vanishing in others (dimensional, $\zeta$ function regularizations). But as the result is proportional to the Seeley-DeWitt coefficient $A_{1}=\beta \mathcal{A}_{H} C_{2}(\beta)$, it can be renormalized away into the bare gravitational action. This fact ensures equivalence of different regularizing functions.
[1] G. 't Hooft, Nucl. Phys. B256, 727 (1985).

[2] L. Susskind and J. Uglum, Phys. Rev. D 50, 2700 (1994).

[3] C. Callan and F. Wilczek, Phys. Lett. B 333, 55 (1994).

[4] J. S. Dowker, Class. Quantum Grav. 11, L55 (1994).

[5] G. Cognola, K. Kirsten, and L. Vanzo, Phys. Rev. D 49, 1029 (1994).

[6] S. N. Solodukhin, Phys. Rev. D 51, 609 (1995).

[7] D. Kabat, Nucl. Phys. B453, 281 (1995).

[8] S. Carlip and C. Teitelboim, Class. Quantum Grav. 12, 1699 (1995).

[9] J.-G. Demers, R. Lafrance, and R. C. Myers, Phys. Rev. D 52, 2245 (1995).

[10] F. Larsen and F. Wilczek, Nucl. Phys. B458, 249 (1996).

[11] D. V. Fursaev and S. N. Solodukhin, Phys. Lett. B 365, 51 (1996).

[12] S. Zerbini, G. Cognola, and L. Vanzo, Phys. Rev. D 54, 2699 (1996).
[13] V. P. Frolov, D. V. Fursaev, and A. I. Zelnikov, Phys. Rev. D 54, 2711 (1996).

[14] E. Elizalde and A. Romeo, Phys. Rev. D 54, 6555 (1996).

[15] M. Hotta, T. Kato, and K. Nagata, Class. Quantum Grav. 14, 1917 (1997).

[16] V. P. Frolov, D. V. Fursaev, and A. I. Zelnikov, Nucl. Phys. B486, 339 (1997).

[17] V. P. Frolov and D. V. Fursaev, Class. Quantum Grav. 15, 2041 (1998).

[18] J. L. F. Barbon, Phys. Rev. D 50, 2712 (1994).

[19] R. Emparan, Phys. Rev. D 51, 5716 (1995).

[20] D. Iellici, Ph.D. thesis, University of Trento, 1998, gr-qc/9805058.

[21] D. Iellici, Class. Quantum Grav. 14, 3287 (1997).

[22] H. S. Carslaw, Proc. London Math. Soc. 18, 291 (1919).

[23] J. S. Dowker, Phys. Rev. D 36, 3095 (1987).

[24] D. V. Fursaev, Class. Quantum Grav. 11, 1431 (1994). 
[25] J. Cheeger, J. Diff. Geom. 18, 575 (1983).

[26] V. Moretti, Phys. Rev. D 56, 7797 (1997).

[27] A. Romeo, Class. Quantum Grav. 13, 2797 (1996).

[28] F. Antonsen and K. Bormann, hep-th/9702009.

[29] S. N. Solodukhin, Phys. Rev. D 51, 618 (1995).

[30] E. S. Moreira, Jr. (in preparation).

[31] E. S. Moreira, Jnr., Nucl. Phys. B451, 365 (1995).

[32] A. P. Prudnikov, Yu. A. Brychkov, and O. I. Marichev, Integrals and Series (Gordon and Breach, Amsterdam, 1986), Vol. 2.

[33] L.J. Slater, Generalized Hypergeometric Functions (Cambridge University Press, Cambridge, England, 1966).
[34] I. Brevik, G. E. A. Matsas, and E. S. Moreira, Jr., Phys. Rev. D 58, 027502 (1998).

[35] A. A. Bytsenko, G. Cognola, and S. Zerbini, Nucl. Phys. B458, 267 (1996).

[36] J. S. Dowker and R. Critchley, Phys. Rev. D 13, 3224 (1976).

[37] R. D. Ball, Phys. Rep. 182, 1 (1989).

[38] A. A. Bytsenko, G. Cognola, L. Vanzo, and S. Zerbini, Phys. Rep. 266, 1 (1996).

[39] N. D. Birrel and P.C.W. Davies, Quantum Fields in Curved Space (Cambridge University Press, Cambridge, England, 1982). 